\title{
Cooperative Communications Protocol for Multiuser OFDM Networks
}

\author{
W. Pam Siriwongpairat, Member, IEEE, Ahmed K. Sadek, Member, IEEE, and K. J. Ray Liu, Fellow, IEEE
}

\begin{abstract}
This paper proposes a relay-based scheme where a fixed number of relays are utilized to design an OFDM cooperative protocol. By exploiting limited feedback from the destination terminal (central node) such that each relay is able to help forward information of multiple sources in one OFDM symbol, the proposed cooperative protocol not only achieves full diversity but also efficiently utilizes available bandwidth. A practical relay assignment scheme is also proposed to specify the pairing of sources and relays in cooperative networks. The outage probability analysis of the proposed cooperative protocol is provided, and the analytical results are validated via simulations. Moreover, a closed-form lower bound on the outage probability of any relay-assignment schemes is established to provide a performance benchmark of the proposed cooperative protocol. Based on the outage probability analysis, the optimum relay location for the proposed relay-assignment scheme is determined. Both theoretical and simulation results show that in WLAN scenario the proposed cooperative protocol can achieve significant coverage extension compared to the direct transmission.
\end{abstract}

Index Terms-OFDM, cooperative protocols, wireless LAN, bandwidth efficient, multiuser, and outage probability.

\section{INTRODUCTION}

$\mathbf{C}$ OOPERATIVE diversity has recently emerged as a promising alternative to combat fading in wireless channels. The basic idea is that users or nodes in a wireless network share their information and transmit cooperatively as a virtual antenna array, thus providing diversity without the requirement of additional antennas at each node. In [1], the authors proposed various cooperative strategies including fixed relaying (e.g. amplify-and-forward and decode-and-forward), selection relaying, and incremental relaying schemes. User cooperation diversity was proposed in [2]. In [3], symbolerror-rate performance analysis and optimal power allocation in a single relay network were considered. A comprehensive treatment of diverse aspects of cooperative communications and networking can be found in [4].

In broadband communications, orthogonal frequency division multiplexing (OFDM) is an effective means to capture multipath energy, mitigate the intersymbol interferences, and offer high spectral efficiency. OFDM is used in many communications systems, e.g., wireless local area networks (WLANs) and wireless personal area networks (WPANs). To improve the performance of OFDM systems, the fundamental concept

Manuscript received December 22, 2006; revised August 14, 2007; accepted October 12, 2007. The associate editor coordinating the review of this paper and approving it for publication was A. Yener.

W. P. Siriwongpairat is with Meteor Communications Corporation, Kent, WA 98032 (e-mail: PSiriwongpairat@meteorcomm.com).

A. K. Sadek is with Corporate Research and Development, Qualcomm Incorporated, San Diego, CA 92121 (e-mail: asadek@qualcomm.com).

K. J. R. Liu is with the Department of Electrical and Computer Engineering and Institute for Systems Research, University of Maryland, College Park, MD 20742 USA (e-mail: kjrliu@eng.umd.edu).

Digital Object Identifier 10.1109/TWC.2008.061106. of cooperative diversity can be applied. Nevertheless, special modulations/cooperation strategies are needed to efficiently exploit the available multiple carriers. In [5], an oversampling technique is used in combination with the intrinsic properties of OFDM symbols to provide efficient resource utilization. An application of space-time cooperation in OFDM systems was investigated in [6]. In [7], pairing of users and level of cooperation are jointly determined to minimize overall transmitted power of OFDM system. Most of the existing works are based on fixed relaying protocols, in which the relays always repeat the source information. Moreover, these works rely on an assumption of fixed channel variances which implies a fixed network topology and fixed source-relay pairs.

In this paper, we propose an OFDM cooperative protocol that improves spectral efficiency over those based on fixed relaying protocols while achieving the same performance of full diversity. By exploiting limited feedback from the destination node, the proposed protocol allows each relay to help forward information of multiple sources in one OFDM symbol. We also propose a practical relay-assignment scheme for implementing the proposed cooperative protocol in OFDM networks. Outage probability is provided as a performance measure of the proposed protocol. The analysis takes into consideration the random users' spatial distribution and the associated propagation path losses between transmit-receive nodes. A closed-form lower bound on the outage probability of any relay-assignment schemes is established as a performance benchmark. Based on the outage probability analysis, we are able to determine the optimum relay locations for the proposed relay-assignment scheme. Simulation results under WLAN scenario are shown to validate our proposed cooperative protocol and support the theoretical analysis.

\section{SYSTEM MOdeL}

We consider an OFDM wireless network such as a WLAN or a WPAN with a circular cell of radius $\rho$. The cell contains one central node and multiple users, each communicating with the central node. The central node can be a base station or an access point in case of the WLAN, and it can be a piconet coordinator in case of the WPAN. Suppose the central node is located at the center of the cell, and $K$ users are uniformly located within the cell. Then, the user's distance $D_{s, d}$ from the central node has the probability density function (PDF): $p_{D_{s, d}}(D)=2 D / \rho^{2}, 0 \leq D \leq \rho$, and the user's angle is uniformly distributed over $[0,2 \pi)$. We assume that each node is equipped with single antenna, and its transmission is constrained to half-duplex mode [1]. We consider an uplink scenario where all users transmit their information to the central node. Channel access within the cell is based on 
orthogonal multiple access mechanism as used in many current OFDM wireless networks.

The received signal at subcarrier $n$ of destination $d$ from source user $s$ can be modeled as

$$
y_{s, d}(n)=\sqrt{P_{n c} \kappa D_{s, d}^{-\nu}} H_{s, d}(n) x_{s}(n)+z_{s, d}(n),
$$

where $P_{n c}$ is the transmitted power in each subcarrier at the source in non-cooperative mode, $x_{s}(n)$ denotes an information symbol to be transmitted from the source $s$ at subcarrier $n$, $z_{s, d}(n)$ is an additive white Gaussian noise with zero mean and variance $N_{0}$, and $H_{s, d}(n)$ is the frequency response of the channel at the $n^{\text {th }}$ subcarrier. Consider OFDM with $N$ subcarriers and assume that the length of cyclic prefix in the OFDM symbol is longer than the channel delay spread, then we can express the channel frequency response as $H_{s, d}(n)=\sum_{l=0}^{L-1} \alpha_{s, d}(l) \exp \left(-j 2 \pi n \tau_{s, d}(l) / N\right)$, where $L$ denotes the number of independent delay paths, $\tau_{s, d}(l)$ is the delay of the $l^{\text {th }}$ path, and $\alpha_{s, d}(l)$ is modeled as circularly symmetric complex Gaussian random variable with zero mean and unit variance. In (1), $\kappa$ is a constant whose value depends on the propagation environment and antenna design, $\nu$ is the propagation loss factor, and $D_{s, d}$ represents the distance between source node $s$ and destination node $d$. The parameters $P_{n c}, \kappa$, and $\nu$ are assumed to be the same for all users.

Let each information symbol have unit energy. For a given distance $D_{s, d}$ between the source and the destination, the received signal-to-noise ratio (SNR) for the $n^{t h}$ subcarrier can be given by

$$
\zeta_{s, d}^{(n)}=P_{n c} \kappa D_{s, d}^{-\nu}\left|H_{s, d}(n)\right|^{2} / N_{0} .
$$

In this paper, we characterize the system performance in terms of the outage probability which is determined by the probability that the instantaneous channel parameters cannot support the required data rate [9]. In case of direct transmission between two nodes that are $D_{s, d}$ apart, the maximum average mutual information in a subcarrier $n$, which is achieved for the independent and identically distributed zeromean circularly symmetric complex Gaussian inputs, is given by $I^{(n)}\left(D_{s, d}\right)=\log \left(1+\zeta_{s, d}^{(n)}\right)$. Let $R$ denote a target rate for each subcarrier, then the probability that a subcarrier is in outage can be given by

$$
\mathrm{P}_{\text {out }}^{D}\left(D_{s, d}\right)=\operatorname{Pr}\left(I^{(n)}\left(D_{s, d}\right) \leq R\right)=\operatorname{Pr}\left(\zeta_{s, d}^{(n)} \leq 2^{R}-1\right) .
$$

If an outage occurs in a subcarrier, then the transmitted information in that subcarrier is considered loss. Otherwise, the receiver is assumed to be able to decode the received information with negligible probability of error.

\section{Proposed Cooperative Protocol And RELAY-ASSIGNMENT SCHEME}

Consider a cooperation scenario where each source can employ a relay to forward its information to the destination. The proposed cooperative protocol builds upon the incremental relaying protocol [1], which exploits a bit feedback from the destination that indicates the success or failure of the direct transmission. The proposed protocol consists of two phases as follows.

In Phase 1, each user transmits its packet to the destination (central node) and the packets are also received at the relay.

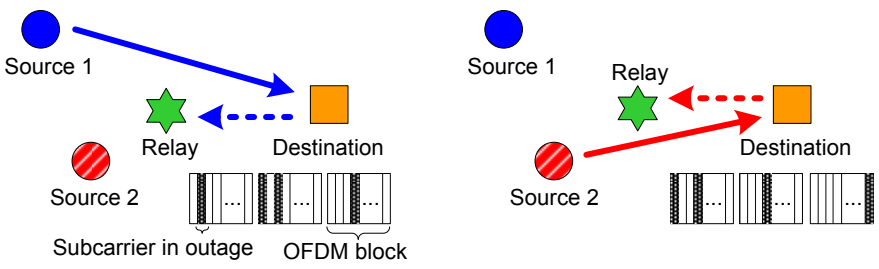

(a) Phase 1: Source transmits information to the destination

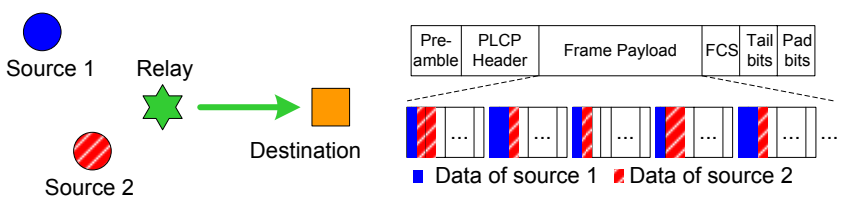

(b) Phase 2: Relay forwards source information to the destination

Fig. 1: Illustrations of the proposed cooperative protocol for OFDM system with 2 users and 1 relay.

After receiving the user's packet, the destination performs channel estimation using the OFDM pilot symbols in the packet header. Based on the estimated channel coefficients, the destination is able to specify which subcarrier symbols are not received successfully (i.e., those in the subcarriers of which the combined SNRs fall below the required SNR threshold), and then broadcasts the indices of the subcarriers carrying those symbols. Such feedback enables the assigned relay to help forward the source information only when necessary.

In Phase 2, the relay decodes the source symbols that are unsuccessfully received at the destination via direct transmission, and then forwards the decoded information to the destination. The relay will only decode the source symbol that the relay is capable of capturing, i.e., when the received SNR at the relay is above the target threshold. Since it is unlikely that all subcarrier symbols are sent unsuccessfully by the source, the proposed protocol makes efficient use of the available bandwidth by allowing the relay to help forward the information of multiple source users in one OFDM block. Specifically, suppose a relay is assigned to help $k$ users, then after all $k$ users transmit their packets in Phase 1, the relay sends in Phase 2 an additional packet containing these users' symbols that are not captured at the destination in Phase 1. In order for the destination to know which relay subcarriers carry information of which users, the relay can send an additional overhead together with the source symbols. Or the relay can use a fixed subcarrier assignment scheme such that that the destination can specify the relay subcarrier assignment without the use of additional overhead. For instance, if $n_{i}$ subcarriers of user $i$ are in outage, then in Phase 2, the relay can use the first $n_{1}$ subcarriers to transmit the data of user 1 , the next $n_{2}$ subcarriers to transmit the data of user 2 , and so on. The fixed subcarrier assignment scheme requires no additional overhead, but it does not fully utilize the resources when both relay and destination cannot decode the source symbols; nevertheless, this event occurs with small probability. Figure 1 illustrates as example of the signal transmissions of the proposed protocol with fixed subcarrier assignment scheme for an OFDM system with 2 source users and 1 relay. Figures 1(a) and 1(b) depict transmission in Phase 1 and Phase 2, respectively.

In Phase 1 , the received signals at the destination and the 


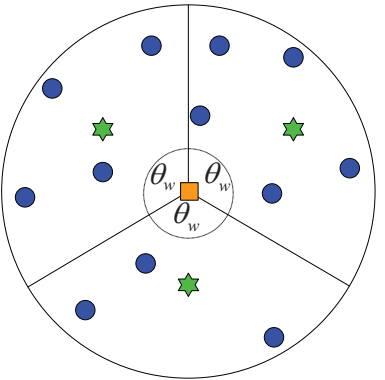

(a) A cell with 3 relays

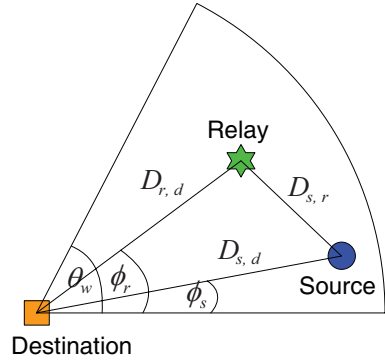

(b) One sector
Fig. 2: An example of relay assignment for a multiuser OFDM system.

relay are $y_{s, d}(n)=\sqrt{P_{c o} \kappa D_{s, d}^{-\nu}} H_{s, d}(n) x_{s}(n)+z_{s, d}(n)$ and $y_{s, r}(n)=\sqrt{P_{c o} \kappa D_{s, r}^{-\nu}} H_{s, r}(n) x_{s}(n)+z_{s, r}(n)$, respectively, where $P_{c o}$ is the transmitted power in the cooperative mode. In Phase 2, the signal received at the destination from the relay is $y_{r, d}(n)=\sqrt{P_{c o} \kappa D_{r, d}^{-\nu}} H_{r, d}(n) \tilde{x}_{s}(n)+z_{r, d}(n)$, where $\tilde{x}_{s}(n)$ denotes the source symbols that are not captured by the destination in Phase 1.

To decide the pairing of sources and relays, we propose in this subsection a practical relay assignment scheme for cooperative OFDM networks. We focus on a fixed-relaylocation scenario in which a certain number of relays are installed in fixed locations in the network. Let $W$ denote the number of relays in a circular cell with radius $\rho$, as illustrated in Figure 2(a). Then, the relay assignment scheme is as follows:

- The cell is equally divided into $W$ sectors, each with central angle $2 \pi / W$. One relay is assigned to help users within each sector.

- In each sector, the relay is placed at an optimum relay location which minimizes the outage probability for all possible source-destination pairs within the sector.

The optimum relay location will be determined in Section IV-C.

\section{Performance Analysis}

In this section, we first analyze the outage probability of the proposed cooperative protocol based on fixed subcarrier assignment scheme, then provide its closed-form lower bound, and finally determine the optimum relay location.

\section{A. Outage Probability}

In the non-cooperative protocol, the channel resources (time and frequency) are divided among $K$ users. In the proposed cooperative protocol, on the other hand, $W$ relays are also utilized, so the same channel resources are divided among $K+W$ users. Thus, for the proposed cooperative protocol, the mutual information in each subcarrier is given by $K /(K+W) I^{(n)}\left(D_{i, j}\right)$, and the event that a subcarrier in $i-j$ transmit-receive link is in outage is $\frac{K}{K+W} I^{(n)}\left(D_{i, j}\right) \leq R$, which corresponds to the event

$$
\left|H_{i, j}(n)\right|^{2} \leq \frac{\left(2^{(K+W) R / K}-1\right) N_{0}}{\kappa P_{c o}} D_{i, j}^{-\nu} \triangleq \beta D_{i, j}^{-\nu} .
$$

Under the proposed cooperative protocol, the average probability that a subcarrier is in outage can be expressed as

$$
\mathrm{P}_{\text {out }}=\left.\sum_{k=0}^{K} \mathrm{P}_{\text {out }}\right|_{k} \operatorname{Pr}(k \text { users in the sector }),
$$

where $\left.\mathrm{P}_{\text {out }}\right|_{k}$ denotes the outage probability per subcarrier given that $k$ users are in the sector. With $W$ sectors in the cell and the assumption that the users are uniformly located in the cell, the chance that a user is located in a specific sector is given by $1 / W$. Accordingly, the probability that $k$ users are located in one sector follows the binomial distribution as

$$
\begin{aligned}
\operatorname{Pr}(k \text { users in the sector }) & =\left(\begin{array}{c}
K \\
k
\end{array}\right)\left(\frac{1}{W}\right)^{k}\left(1-\frac{1}{W}\right)^{K-k} \\
& \triangleq c(k), \quad k \in\{0,1, \ldots, K\} .
\end{aligned}
$$

The conditional outage probability per subcarrier, $\left.\mathrm{P}_{\text {out }}\right|_{k}$, can be determined as follows. In case that the relay has available resources to help forward the source information, the outage event in subcarrier $n$ is equivalent to the event

$$
\begin{aligned}
& \left(\left\{I^{(n)}\left(D_{s, d}\right) \leq \tilde{R}\right\} \cap\left\{I^{(n)}\left(D_{s, r}\right) \leq \tilde{R}\right\}\right) \cup \\
& \left(\left\{I^{(n)}\left(D_{s, d}\right) \leq \tilde{R}\right\} \cap\left\{I^{(n)}\left(D_{r, d}\right) \leq \tilde{R}\right\} \cap\right. \\
& \left.\left\{I^{(n)}\left(D_{s, r}\right)>\tilde{R}\right\}\right),
\end{aligned}
$$

where $\tilde{R}=(K+W) R / K$. The first term in the union of (7) corresponds to the event that both the source-destination link and source-relay link are in outage, while the second term corresponds to the event that both the source-destination link and relay-destination link are in outage while the source-relay link is not. According to (4) and Rayleigh distribution, we have $\operatorname{Pr}\left(\left\{I^{(n)}\left(D_{i, j}\right) \leq \tilde{R}\right\}\right)=\operatorname{Pr}\left(\left|H_{i, j}(n)\right|^{2} \leq \beta D_{i, j}^{\nu}\right)=$ $1-\exp \left(-\beta D_{i, j}^{\nu}\right)$, where $\beta$ is defined in (4). Note that the events in the union in (7) are mutually exclusive. Hence, the conditional outage probability under the cooperative mode can be determined as

$$
\begin{aligned}
\mathrm{P}_{\text {out }}^{C}= & {\left[1-\exp \left(-\frac{\left(2^{\tilde{R}}-1\right) N_{0} D_{s, d}^{\nu}}{\kappa P_{c o}}\right)\right] \times } \\
& {\left[1-\exp \left(-\frac{\left(2^{\tilde{R}}-1\right) N_{0}}{\kappa P_{c o}}\left(D_{s, r}^{\nu}+D_{r, d}^{\nu}\right)\right)\right] . }
\end{aligned}
$$

In case that the relay does not have resource to help forward the source information, the outage event in subcarrier $n$ is equivalent to that of direct transmission between the source and destination, which is given by

$$
\begin{aligned}
\mathrm{P}_{\text {out }}^{D} & =\operatorname{Pr}\left(I^{(n)}\left(D_{s, d}\right) \leq \tilde{R}\right) \\
& =1-\exp \left(-\frac{\left(2^{(K+W) R / K}-1\right) N_{0} D_{s, d}^{\nu}}{\kappa P_{c o}}\right) .
\end{aligned}
$$

Given $k$ users in the sector, the conditional outage probability per subcarrier can be obtained from (8) and (9) as

$$
\left.\mathrm{P}_{\text {out }}\right|_{k}=\mathrm{P}_{\text {out }}^{C}\left(1-\left.Q\right|_{k}\right)+\left.\mathrm{P}_{\text {out }}^{D} Q\right|_{k} \text {. }
$$

In (10), $\left.Q\right|_{k}$ is defined as the probability that a source subcarrier that is in outage is not helped by the relay, under the condition that there are $k$ users in the sector. In other words, the relay uses all of its resources to help forward the $k$ users' information carried in other subcarriers. The physical meaning 
of (10) is that a diversity gain of two is achieved when the relay has available resource to help forward the source information, which occurs with probability $\left.Q\right|_{k}$, whereas the diversity gain reduces to one when all the resources (allocated subcarriers) at the relay are fully used.

The probability $\left.Q\right|_{k}$ can be determined as follows. Consider a sector with $k$ users, each transmitting information in $N$ subcarriers during one OFDM symbol period. Equivalently, the total number of $k N$ subcarriers (in $k$ OFDM symbol periods) are used to transmit the information from all $k$ users in the sector. Assume that the subcarriers in outage are helped by the relay with equal probability. Then, given that $x$ out of $k N$ subcarriers are in outage, the probability that a subcarrier in outage is not helped by the relay is $(x-N) / x$ for $N+1 \leq x \leq k N$. Thus, the probability $\left.Q\right|_{k}$ can be obtained as

$$
\left.Q\right|_{k}=\sum_{x=N+1}^{k N} \frac{x-N}{x} q(x),
$$

where $q(x) \triangleq \operatorname{Pr}(x$ out of $k N$ subcarriers in outage $)$. Since each subcarrier is in outage with probability $\mathrm{P}_{\text {out }}^{D}$, the probability that $x$ out of $k N$ subcarriers are in outage can be approximated by ${ }^{1}$

$$
q(x)=\left(\begin{array}{c}
k N \\
x
\end{array}\right)\left(\mathrm{P}_{\text {out }}^{D}\right)^{x}\left(1-\mathrm{P}_{\text {out }}^{D}\right)^{k N-x} .
$$

Substituting (9) and (12) into (11), we obtain the probability $\left.Q\right|_{k}$ as

$$
\left.Q\right|_{k}=\sum_{x=N+1}^{k N} \frac{x-N}{x}\left(\begin{array}{c}
k N \\
x
\end{array}\right)\left(1-e^{-\beta D_{s, d}^{\nu}}\right)^{x}\left(e^{-\beta D_{s, d}^{\nu}}\right)^{k N-x} .
$$

From (13) and (10), we can determine the conditional outage probability of the proposed scheme as

$$
\begin{aligned}
\left.\mathrm{P}_{\text {out }}\right|_{k}= & \left(1-e^{-\beta D_{s, d}^{\nu}}\right)\left(1-e^{-\beta\left(D_{s, r}^{\nu}+D_{r, d}^{\nu}\right)}\right)\left(1-\left.Q\right|_{k}\right)+ \\
& \left.\left(1-e^{-\beta D_{s, d}^{\nu}}\right) Q\right|_{k} .
\end{aligned}
$$

Substituting (6) and (14) into (5), the outage probability of the proposed scheme can be obtained as

$$
\begin{aligned}
\mathrm{P}_{\text {out }}= & \left(1-e^{-\beta D_{s, d}^{\nu}}\right)\left[1-e^{-\beta\left(D_{s, r}^{\nu}+D_{r, d}^{\nu}\right)}\left(1-g\left(D_{s, d}\right)\right)\right] ; \\
g\left(D_{s, d}\right)= & \sum_{k=1}^{K} c(k) \sum_{x=N+1}^{k N} \frac{x-N}{x}\left(\begin{array}{c}
k N \\
x
\end{array}\right) \times \\
& \left(1-e^{-\beta D_{s, d}^{\nu}}\right)^{x}\left(e^{-\beta D_{s, d}^{\nu}}\right)^{k N-x}
\end{aligned}
$$

It is worth noting that when spectral efficiency $(R)$ goes to infinity, direct transmission outperforms cooperative communications. As the number of users $(K)$ increases, for a fixed number of relays, the performance of the proposed cooperative scheme approaches that of direct transmission.

Finally, we determine the average outage probability by

\footnotetext{
${ }^{1}$ In (12), it is difficult, if not impossible, to derive a closed form formulation that includes all possible combinations of the users' locations. For analytical tractability of the analysis, we resort to an approximate probability which is based on an assumption that all the $x$ subcarriers are in outage with the same probability $\mathrm{P}_{\mathrm{out}}^{D}$. This approximation would be effective for small cell sizes where path loss is not the dominant fading effect. As we will show in Section $\mathrm{V}$, the analytical outage probability obtained from this approximation is very close to the simulation results.
}

averaging (15) over the distribution of the user's distance as follows. Without loss of generality, we consider a sector as shown in Figure 2(b), in which where the relay is located at $D_{r, d} e^{\mathbf{j} \phi_{r}}$ and a source user is located at $D_{s, d} e^{\mathbf{j} \phi_{s}}$ $\left(0 \leq \phi_{r}, \phi_{s} \leq \theta_{w}\right)$. The distance between the source and the relay can be expressed as

$$
\begin{aligned}
D_{s, r} & =\left[D_{s, d}^{2}+D_{r, d}^{2}-2 D_{s, d} D_{r, d} \cos \left(\phi_{r}-\phi_{s}\right)\right]^{\frac{1}{2}} \\
& \triangleq f\left(D_{s, d}, \phi_{s}\right) .
\end{aligned}
$$

Assuming that users are uniformly distributed within the cell, the PDF of the user's distance $D$ from the destination conditioned that the user is located in the sector can be given by

$$
p_{D}\left(D \mid 0 \leq \phi_{s} \leq \theta_{W}\right)=\frac{2 D}{W \rho^{2}}, \quad 0 \leq D \leq \rho .
$$

Therefore, given specific relay locations, the average outage probability of the proposed cooperative protocol can be obtained as

$$
\begin{aligned}
\overline{\mathrm{P}}_{\text {out }}=\frac{1}{\pi \rho^{2}} \int_{0}^{\rho} \int_{0}^{\frac{2 \pi}{W}}\left(1-r\left(D_{s, d}, \phi_{s}\right)\right) \times \\
D_{s, d}\left(1-e^{-\beta D_{s, d}^{\nu}}\right) d \phi_{s} d D_{s, d},
\end{aligned}
$$

where

$$
r\left(D_{s, d}, \phi_{s}\right) \triangleq e^{-\beta\left(f^{\nu}\left(D_{s, d}, \phi_{s}\right)+D_{r, d}^{\nu}\right)}\left(1-g\left(D_{s, d}\right)\right) .
$$

From (19), we can clearly see that the performance of the proposed cooperative protocol depends on the number of relays in the cell and how each relay are assigned to help the source users. To get more insights of the cooperation systems, we provide the lower bound on the outage probability of the proposed cooperative protocol and the performance of the proposed relay-assignment scheme in the following subsections.

\section{B. Performance Lower Bound}

We exploit a hypothetical Genie-aided relay-assignment scheme [11] to find a lower bound on the outage probability of any practical relay-assignment schemes as follows. The Genieaided relay-assignment scheme assumes that the assigned relay for any source in the network is located in the optimum location that minimize the outage probability for the fixed source-destination pair.

Observe that if the relay can be placed anywhere in the cell, the optimum relay location for a source-destination pair is on the line joining the source and destination. Accordingly, the optimum distance between the relay and destination can be written as $D_{s, r}=D_{s, d}-D_{r, d}$. Thus, from (8), the optimum relay location for a source-destination pair can be obtained by solving

$$
\begin{gathered}
D_{r, d}^{*}=\arg \min _{D_{r, d}} 1-e^{-\beta\left(\left(D_{s, d}-D_{r, d}\right)^{\nu}+D_{r, d}^{\nu}\right)}\left(1-g\left(D_{s, d}\right)\right) \\
\text { subject to } 0 \leq D_{r, d} \leq D_{s, d} .
\end{gathered}
$$

The optimization problem above is equivalent to find $D_{r, d}$ that minimizes $\left(D_{s, d}-D_{r, d}\right)^{\nu}+D_{r, d}^{\nu}$. Thus, it can be analytically solved simply, and the optimum solution can be shown to be $D_{r, d}^{*}=D_{s, d} / 2$, i.e., the optimum relay location for a specific 
source-destination pair is at the mid point of the line joining the source and the destination.

Using the Genie-aided relay-assignment scheme and the optimum relay location $D_{r, d}^{*}$, we can determine the lower bound on the outage probability as follows. Substitute $D_{r, d}^{*}$ and $D_{s, r}^{*}=D_{s, d}-D_{r, d}^{*}=D_{s, d} / 2$ into the outage probability formulation in (19), then the outage probability of the proposed cooperative protocol can be lower bounded by

$$
\mathrm{P}_{\text {out }} \geq\left(1-e^{-\beta D_{s, d}^{\nu}}\right)\left[1-e^{-\beta D_{s, d}^{\nu} /\left(2^{\nu-1}\right)}\left(1-g\left(D_{s, d}\right)\right)\right]
$$

Averaging (21) over all users' possible locations, we have

$$
\begin{aligned}
\overline{\mathrm{P}}_{\mathrm{Out}} & \geq \frac{2}{\rho^{2}} \int_{0}^{\rho} D_{s, d}\left(1-e^{-\beta D_{s, d}^{\nu}}\right)\left[1-e^{-\beta D_{s, d}^{\nu} / 2^{\nu-1}}\right] d D_{s, d} \\
& +\frac{2}{\rho^{2}} \sum_{k=0}^{K} c(k) \sum_{x=N+1}^{k N} \frac{x-N}{x}\left(\begin{array}{c}
k N \\
x
\end{array}\right) \\
& \times \int_{0}^{\rho} e^{-\beta D_{s, d}^{\nu}\left(k N-x+\frac{1}{2^{\nu-1}}\right)}\left(1-e^{\left.-\beta D_{s, d}^{\nu}\right)^{x+1}} D_{s, d} d D_{s, d} .\right.
\end{aligned}
$$

At high SNR, the effect of the second term in (22) on the outage probability is insignificant. Thus, neglecting the second term in (22), the average outage probability of the proposed protocol can be lower bounded by

$$
\begin{aligned}
\overline{\mathrm{P}}_{\text {out }} \geq & +\frac{2}{\nu \rho^{2}}\left(\frac{1}{\beta\left(1+2^{1+\nu}\right)}\right)^{\frac{2}{\nu}} \Upsilon\left(\frac{2}{\nu}, \beta\left(1+2^{1+\nu}\right) \rho^{\nu}\right) \\
& -\frac{2}{\nu \rho^{2}}\left(\frac{1}{\beta}\right)^{\frac{2}{\nu}} \Upsilon\left(\frac{2}{\nu}, \beta \rho^{\nu}\right)-\frac{2}{\nu \rho^{2}}\left(\frac{1}{\beta\left(1+2^{1+\nu}\right)}\right)^{\frac{2}{\nu}} \\
& \times \Upsilon\left(\frac{2}{\nu}, 2^{1-\nu} \beta \rho^{\nu}\right) .
\end{aligned}
$$

\section{Optimum Relay Location}

Based on the average outage probability (19), we determine in this subsection the optimum relay location in each sector for a cell with $W$ sectors. Since the users are uniformly located in the cell, one can show that the optimum relay location is on the line that divides the central angle $\theta_{w}$ into two equal parts, i.e., the optimum relay angle is $\phi_{r}^{*}=\theta_{w} / 2$. Now, the remaining problem is to determine the optimum relay distance $\hat{D}_{r, d}$. Substitute $\phi_{r}^{*}$ into (19) and take the first derivative of $\overline{\mathrm{P}}_{\text {out }}$ with respect to $D_{r, d}$, then the optimum relay distance $D_{r, d}^{*}$ can be obtained by solving

$$
\begin{gathered}
\int_{0}^{\rho} D_{s, d}\left(1-\exp \left(-\beta D_{s, d}^{\nu}\right)\right)\left(1-g\left(D_{s, d}\right) \int_{0}^{\frac{2 \pi}{W}} \eta\left(D_{s, d}, \phi_{s}\right)\right. \\
\quad \times \exp \left(-\beta\left(f^{\nu}\left(D_{s, d}, \phi_{s}\right)+D_{r, d}^{\nu}\right)\right) d \phi_{s} d D_{s, d}=0, \quad(24)
\end{gathered}
$$

in which $\eta\left(D_{s, d}, \phi_{s}\right)=\left[D_{s, d}^{2}+D_{r, d}^{2}-2 D_{s, d} D_{r, d} \cos (\pi / W-\right.$ $\left.\left.\phi_{s}\right)\right]^{\frac{\nu}{2}-1}\left(D_{r, d}-D_{s, d} \cos \left(\pi / W-\phi_{s}\right)\right)+D_{r, d}^{\nu-1}$.

To get more insights, we also provide here an explicit relay location that achieves close performance to that of optimum relay location. First, we find the average value of all possible users' locations as

$$
\bar{D}_{s, d}=\int_{0}^{\rho} D_{s, d} p_{D_{s, d}}\left(D_{s, d}\right) d D_{s, d}=2 \rho / 3 .
$$

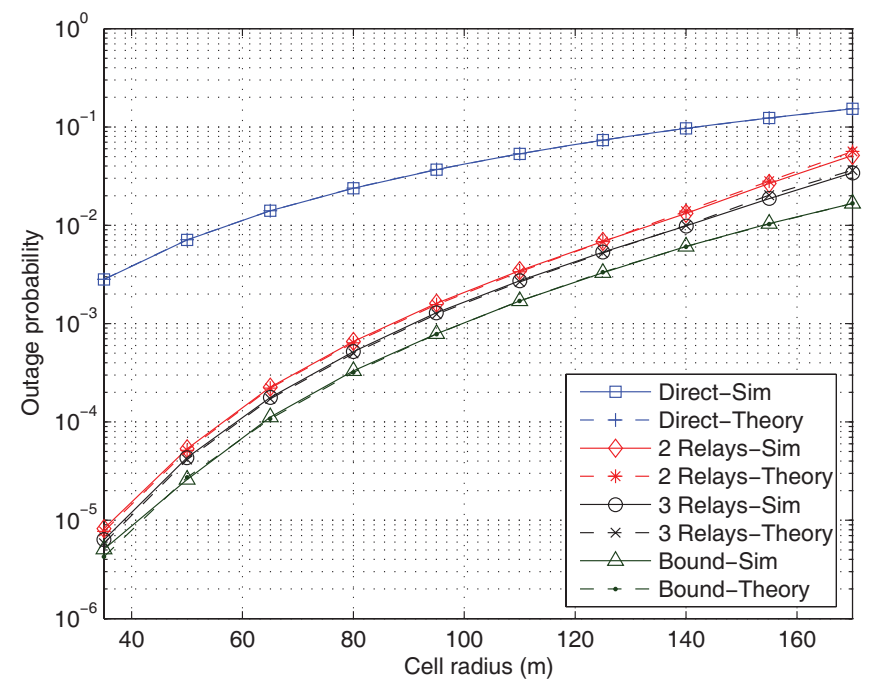

(a) Outage probability versus cell radius

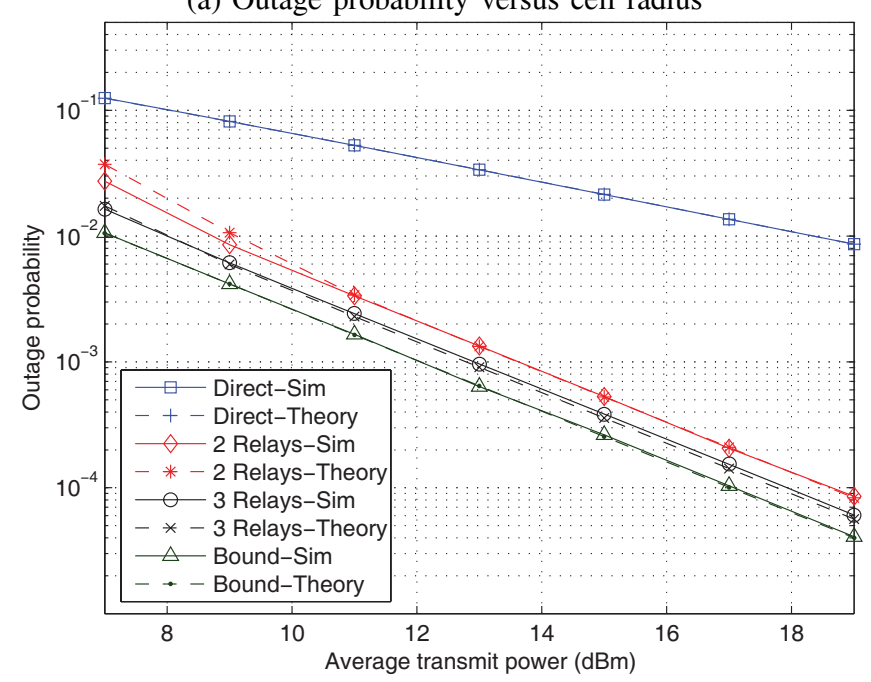

(b) Outage probability versus average transmitted power

Fig. 3: Outage probability performance in WLAN scenario.

From (10) and (25), an approximate relay location can be obtained by solving

$\bar{D}_{r, d}=\arg \max _{0 \leq D_{r, d} \leq \bar{D}_{s, d}} e^{-\beta\left(\left(\bar{D}_{s, d}-D_{r, d}\right)^{\nu}+D_{r, d}^{\nu}\right)}\left(1-g\left(\bar{D}_{s, d}\right)\right)$,

which results in $\bar{D}_{r, d}=\bar{D}_{s, d} / 2=\rho / 3$. The approximate relay location can be shown to achieve very close performance to the optimum relay location.

\section{Simulation Results}

We performed simulation scenarios under WLAN scenario. Each OFDM symbol has 64 subcarriers and the total bandwidth is $20 \mathrm{MHz}$. The target rate is fixed at $R=1 \mathrm{bit} / \mathrm{s} / \mathrm{Hz}$ per user per subcarrier. The propagation loss factor is $\nu=2.6$, and the number of users in the cell is set at 10 users.

Figures 3(a) and 3(b) compare the performance of the proposed cooperative protocol with that of direct transmission scheme and the lower bound. The theoretical performance is plotted along with the simulation curves.

Figure 3(a) depicts the outage probability versus the cell radius. The average transmitted power is fixed at $12 \mathrm{dBm}$. If the outage performance is required to be at most 0.01 , then 
the maximum cell size achieved by the direct transmission scheme is about $60 \mathrm{~m}$. With the proposed cooperative protocol, the maximum cell size of $132 \mathrm{~m}$ and $140 \mathrm{~m}$ can be achieved when 2 and 3 relays are deployed, respectively. The proposed cooperative protocol can increase the cell size by about $130 \%$ in this case. Moreover, the proposed scheme achieves close performance to the lower bound, especially when the cell size is small.

In Figure 3(b), we study the performance gain that can be achieved by the proposed protocol in terms of energy efficiency. The cell radius is fixed at $100 \mathrm{~m}$, and the average transmitted power varies from $7 \mathrm{dBm}$ to $19 \mathrm{dBm}$. If the outage is required to be at most 0.01 , then the direct transmission scheme requires the transmitted power of $18 \mathrm{dBm}$, whereas the proposed scheme requires only about $8 \mathrm{dBm}$; in other words, $10 \mathrm{~dB}$ power saving is achieved. It is worth pointing out that diversity is a high SNR concept and the proposed cooperative scheme is able to achieve the diversity gain of two at practical transmit powers.

In both figures, the theoretical curves closely match to the simulation results, which validates our analysis. Note that the simulation and theoretical results completely match in the direct transmission case.

\section{CONCLUSIONS}

We propose in this paper a bandwidth-efficient cooperative protocol for multiuser OFDM systems. In the proposed protocol, the destination broadcasts subcarrier indices of which the received SNR falls below a specific SNR threshold, and the relay forwards only the source symbols carried in those subcarriers. In this way, the relay can help forward the data of multiple sources in one OFDM symbol, and the proposed protocol greatly improves the spectral efficiency, while still achieving full diversity at high SNR. For practical implementation of the proposed cooperative protocol in OFDM networks, we proposed a relay-assignment scheme in which the cell is divided into sectors and a relay is assigned to help users within the sector. Performance analysis in terms of outage probability is provided. Both analytical and theoretical results show that in WLAN scenario, the proposed cooperative protocol can achieve significant power saving or coverage extension compared to the direct transmission.

\section{REFERENCES}

[1] J. N. Laneman, D. N. C. Tse, and G. W. Wornell, "Cooperative diversity in wireless networks: efficient protocols and outage behavior," IEEE Trans. Inform. Theory, vol. 50, no. 12, pp. 3062-3080, Dec. 2004.

[2] A. Sendonaris, E. Erkip, and B. Aazhang, "User cooperation diversity, part I: system description," IEEE Trans. Commun., vol. 51, no. 11, pp. 1927-1938, Nov. 2003.

[3] W. Su, A. K. Sadek, and K. J. R. Liu, "Cooperative communications in wireless networks: performance analysis and optimum power allocation," Wireless Personal Commun., vol. 44, no. 2, pp. 181-217, Jan. 2008.

[4] K. J. R. Liu, A. K. Sadek, W. Su, and A. Kwasinski, Cooperative Communications and Networking. Cambridge University Press, 2008.

[5] A. Bletsas and A. Lippman, "Efficient collaborative (viral) communication in OFDM based WLANs," in Proc. Inter. Symposium on Advanced Radio Technologies (ISART 2003), Boulder CO, Mar. 2003.

[6] S. Yatawatta and A. P. Petropulu, "A multiuser OFDM system with user cooperation," in Proc. 38th Asilomar Asilomar Conf. on Signals, Systems, and Computers, vol. 1, pp. 319-323, Nov. 2004.

[7] Z. Han, T. Himsoon, W. P. Siriwongpairat, and K. J. R. Liu, "Energyefficient cooperative transmission over multiuser OFDM networks: who helps whom and how to cooperate," in Proc. IEEE Wireless Commun. and Networking Conf., vol. 2, pp. 1030-1035, Mar. 2005.

[8] M. K. Simon and M. S. Alouini, Digital Communication over Fading Channels: A Unified Approach to Performance Analysis. New York: John Wiley and Sons, 2000.

[9] L. H. Ozarow, S. Shamai, and A. D. Wyner, "Information theoretic considerations for cellular mobile radio," IEEE Trans. Veh. Technol., vol. 43, pp. 359-378, May 1994.

[10] J. G. Proakis, Digital Communications, $4^{\text {th }}$ Ed. New York: McGrawHill, 2001.

[11] A. K. Sadek, Z. Han, and K. J. R. Liu, "An efficient cooperation protocol to extend coverage area in cellular networks," in Proc. IEEE Wireless Commun. and Networking Conf., Apr. 2006. 